\title{
Primer hallazgo de Nibilia antilocapra (Stimpson, 1871) (Crustacea: Decapoda: Epialtidae) para Venezuela
}

\author{
First record of Nibilia antilocapra (Stimpson, 1871)(Crustacea: Decapoda: Epialtidae) for Venezuela \\ Iván Hernández-Ávila ${ }^{1}$, Carlos Lira ${ }^{1}$ y Juan Bolaños ${ }^{1}$ \\ ${ }^{1}$ Grupo de Investigación en Carcinología, Escuela de Ciencias Aplicadas del Mar, Universidad de Oriente, Boulevard El Paseo, \\ Edificio IIC, Boca del Río 6304, Isla de Margarita, Estado Nueva Esparta, Venezuela \\ ivanhernavila@yahoo.com
}

\begin{abstract}
Seven specimens of Nibilia antilocapra were collected in Margarita Island, Venezuela. This is the first record of $N$. antilocapra for Venezuela, and for the south Caribbean area. Nibilia antilocapra can be separated from other species of the family Epialtidae by the following features: wide postorbital tooth forming a cup, supraocular eave and postocular cup well
\end{abstract}

\section{Introducción}

La familia Epialtidae es la más diversa de los Majoidea albergando 385 de las 960 especies reconocidas a nivel mundial (Ng et al. 2008). Especies de Majoidea han sido señaladas en numerosos estudios taxonómicos e inventarios faunísticos en aguas someras de Venezuela encontrándose 53 especies, de las cuales 16 pertenecen a Epialtidae; sin embargo, existen muy pocos registros para aquellas especies que habitan a profundidades mayores a 60 m (Rodríguez 1980, Hernández et al. 1992, CarmonaSuárez \& Conde 1996, García et al. 1998, Bolaños et al. 2001, Marcano \& Bolaños 2001, Hernández-Ávila et al. 2007). La subfamilia Pisinae alberga a miembros de la familia Epialtidae que se caracterizan por poseer órbitas incompletas, muy abiertas; con un diente o lóbulo postocular en forma de copa en el cual el ojo, ubicado en un corto pedúnculo, puede retraerse parcialmente; el artejo basal de la antena es ancho en la base, con un diente o espina en el ángulo anterior (Rathbun 1925, Williams 1984, Ng et al. 2008). En el presente artículo se señala la presencia de Nibilia antilocapra para aguas marinas venezolanas, y se describen las principales características morfológicas que distinguen a esta especie.

\section{Material y métodos}

Los especimenes estudiados fueron capturados en distintas localidades de la Isla de Margarita y se encuentran depositados en la colección de crustáceos decápodos del Grupo de Investigación en Carcinología, Universidad de Oriente, Núcleo Nueva Esparta, y otros dos ejemplares se encuentran en el Museo Marino de Margarita (MMM.cr.3148), Venezuela. separated, with a small tooth between them. Carapace pyriform, longer than wide, with several spines of different size on the surface. Chelipeds stouter than the walking leg, the formers diminish gradually in size. Four species of the subfamily Pisinae have been recorded for Venezuela marine waters.

Key words: Brachyura, Majoidea, Caribbean

Las artes de pesca utilizadas para extraer a los crustáceos fueron nasas y filete fondero (González et al. 2006). La especie fue identificada utilizando claves, descripciones e ilustraciones provistas por Rathbun (1925), Williams $(1965,1984)$ y Abele \& Kim (1986). Se tomaron imágenes digitales de alta resolución, mientras que otras estructuras (e.g., gonópodos) fueron dibujadas utilizando una cámara clara acoplada a una lupa esteroscópica, las medidas fueron tomadas con un vernier calibrado.

\section{Resultados y discusión}

Familia Epialtidae MacLeay, 1838

Subfamilia Pisinae Dana, 1851

Género Nibilia A. Milne Edwards, 1878

Nibilia antilocapra (Stimpson, 1871) Fig. 1a-d

Pisa antilocapra Stimpson 1871: 110.

Nibilia antilocapra - Rathbun 1925: 290, láms. 102, 103, 239. Williams 1965: 251 Figs. 230, 233f. Williams 1984: 320, Figs. 254, 259 f.

Nibilia antilocarpa. ${ }^{-} \mathrm{Ng}$ et al. 2008: 104.

Material examinado.- El Tirano, Isla de Margarita $\left(\approx 11^{\circ} 04^{\prime} \mathrm{N} 63^{\circ} 48^{\prime} \mathrm{W}\right), 3$ machos y 2 hembras, capturados con nasas a $50 \mathrm{~m}$ profundidad. La Pared, Península de Macanao, Isla de Margarita $\left(\mathrm{H} \approx 11^{\circ} 05^{\prime} \mathrm{N}-64^{\circ} 16^{\prime} \mathrm{W}\right), 1$ macho (LC=98 mm); 1 hembra ( $\mathrm{LC}=75 \mathrm{~mm})$; capturados con filete fondero a $190 \mathrm{~m}$ de profundidad; colector: Pescadores de la Pared, 25 de mayo de 2006. 


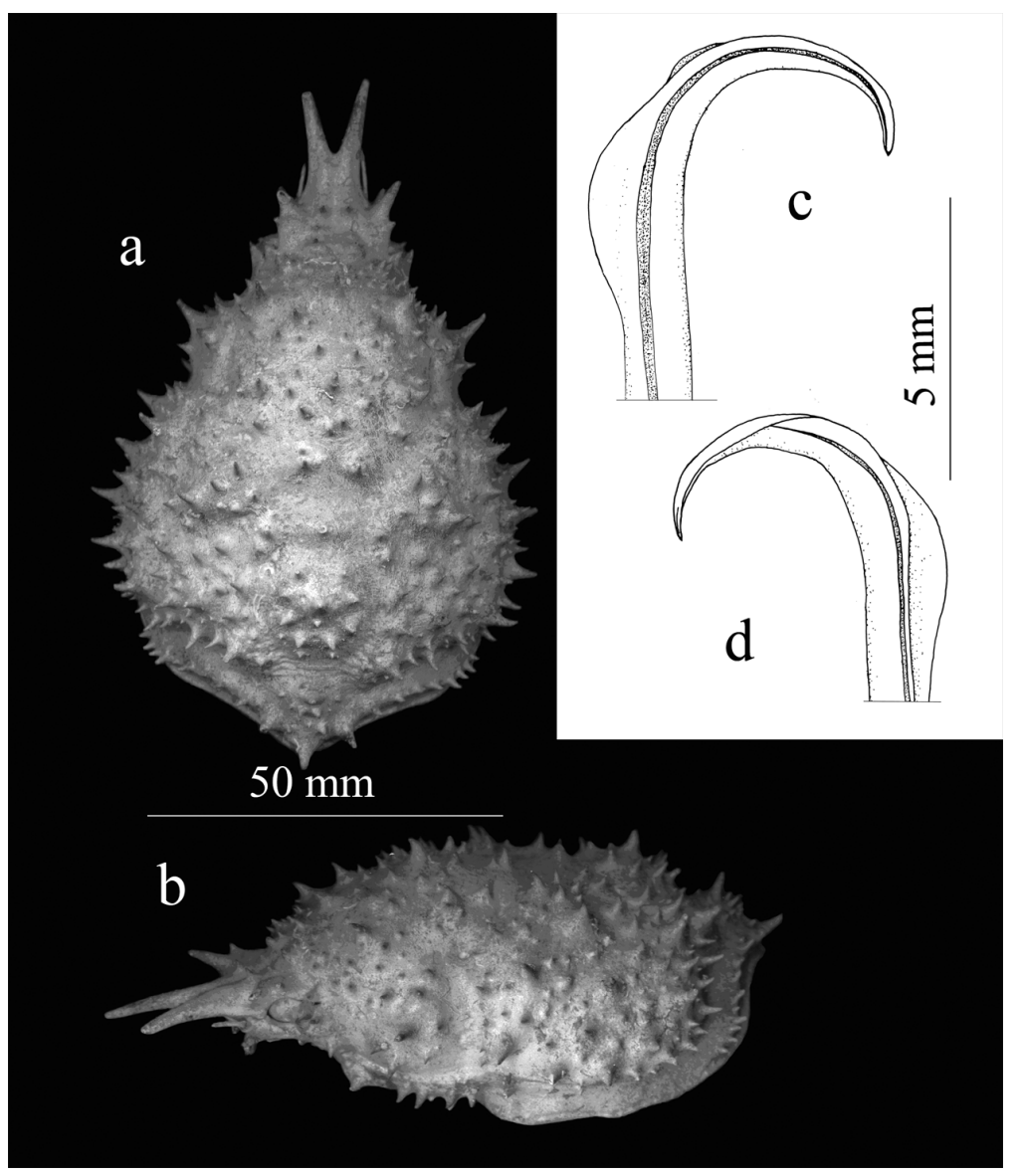

Figure 1

Nibilia antilocapra, a.- caparazón, vista dorsal; b.- caparazón, vista lateral; c.- gonópodo, vista abdominal; d.- gonópodo, vista esternal

Nibilia antilocapra, a.- caparace, dorsal view; b.- carapace, lateral view; c.- gonopod, abdominal view; d.- gonopod, sternal view

Descripción.- Caparazón piriforme, más largo que ancho, espinoso; regiones cardiaca y gástrica con alrededor de 18 espinas de tamaño moderado, las regiones intestinal, branquial y cardiaca se encuentran ocupadas cada una por una espina de mayor tamaño, rodeada de espinas menores dispuestas en círculos, cuatro espinas forman un rombo transverso en la región intestinal. Una serie de espinas robustas se encuentra sobre el margen lateral, siendo la más grande de todas, la ubicada en la región hepática, las espinas postero laterales son menos robustas, y con apariencia más regular. Rostro bifurcado en $2 / 3$ de su longitud, horizontal en vista lateral, longitud $1 / 5$ del largo del caparazón. Espina preorbital dirigida hacia arriba, levemente curvada, casi alcanza la bifurcación del rostro, en la base de ésta existe una pequeña espina, borde supraocular con una espina subtriangular, justo delante de la espina postorbital, artejo basal de la antena con una fuerte espina distolateral y tres espinas de menor tamaño en el borde externo. Pterigostomio ornamentado con dos hileras de espinas.

Los quelípedos del macho son más anchos y largos que las patas caminadoras, la quela se presenta sin espinas, con un diente molariforme en el ápice del dáctilo, al cerrar la quela se forma un hiato entre el dedo fijo y el dáctilo; mero y carpo con tubérculos bajos. Patas caminadoras con tubérculos y espinas leves ordenadas horizontalmente en el mero y el carpo, própodo y dáctilo inermes.

El abdomen de ambos sexos presenta seis segmentos separados más el telson, tres espinas en cada uno de tres primeros segmentos del macho, hembras inermes, gonópodos del macho como se ilustran (Fig. 1c, d). 
Nibilia antilocapra puede ser diferenciada de los demás miembros americanos de la subfamilia Pisinae en poseer un ancho diente postorbital, la proyección supraocular y la copa postocular separadas, con un diente entre estas, caparazón claramente más largo que ancho, con numerosas espinas de tamaño variado, quelípedos más gruesos que las patas caminadoras, disminuyendo estas en tamaño gradualmente en sentido posterior (Rathbun 1925). Esta especie ha sido reportada previamente para aguas de Cabo Hatteras, Florida (localidad tipo), Golfo de México, Saint Vicent y Barbados, a profundidades entre 94 y $252 \mathrm{~m}$ (Rathbun 1925, Williams 1984), siendo el presente hallazgo el límite sur de la especie en el Caribe. Además, existen reportes de esta especie en las afueras de Guyana (Williams 1984) y en Rio Grande do Norte y Rio Grande do Sur, Brasil (Melo 1996).

Los registros de miembros de la subfamilia Pisinae son escasos en Venezuela, Rodríguez (1980) señala la presencia de Libinia ferreirae Brito Capelo, 1871 en aguas del Golfo de Venezuela y el litoral central y Chorinus heros (Herbst, 1790) para la Isla de Margarita; además sugiere la presencia de Herbstia depressa Stimpson, 1860, basado en reportes previos para la isla de Curaçao (Rathbun 1925) ubicada cerca de las costas venezolanas, Marcano \& Bolaños (2001) presuponen la presencia de Notolopas brasiliensis Mier, 1886, basados en el intervalo de distribución reportado por Rathbun (1925), quien indica que la especie se distribuye de Colombia a Brasil, pero no reporta especímenes recolectados en Venezuela; por último Hernández-Ávila et al. (2007) recolectaron un ejemplar de Pelia mutica (Gibbes, 1850) en la isla de Cubagua.

La subfamilia Pisinae posee un gran número de representantes en aguas profundas del Caribe, algunos de ellos de importancia comercial (Rathbun 1925). Es posible que un incremento en los estudios de fauna a grandes profundidades en Venezuela derive en el incremento del número de especies registradas hasta ahora. La ampliación de la distribución de $N$. antilocapra a aguas al sur del Caribe, junto con los reportes previos en Florida, Guyana y Brasil sugiere que la especie posee una distribución continua entre Cabo Hatteras, Florida y Río Grande do Sur (Brasil).

\section{Agradecimientos}

Los autores desean agradecer a los evaluadores por los comentarios sobre el manuscrito y al Departamento de Investigaciones del Museo Marino de Margarita por permitir la revisión de los especímenes allí depositados. Iván Hernández-Ávila posee una subvención de estudios de postgrado a través del FONACIT, contrato 200601239.

\section{Literatura citada}

Abele L \& W Kim. 1986. An illustrated guide to the marine decapod crustaceans of Florida, 760 pp. Florida State University, Tallahasse.

Bolaños J, G Hernández \& C Lira. 2001. Mithraculus cinctimanus Stimpson 1860 y Speloeophorus pontifer (Stimpson 1871) (Crustacea: Decapoda: Brachyura) dos nuevas adiciones a la carcinofauna venezolana. Boletín del Instituto Oceanográfico de Venezuela 39(1-2): 25-31.

Carmona-Suárez C \& J Conde. 1996. Littoral brachyuran crabs (Crustacea: Decapoda) from Falcon, Venezuela, with biogeographical and ecological remarks. Revista Brasilera do Biología 56(4): 725-747.

García L, G Hernández \& J Bolaños. 1998. Anomura y Brachyura de Isla de las Aves. Saber 10(2): 26-31.

González L, E Eslava \& F Guevara. 2006. Catálogo de la pesca artesanal del Estado Nueva Esparta, Venezuela, 222 pp. Instituto de Investigaciones Cientificas, Universidad de Oriente, Cumaná.

Hernández G, L Lares \& J Bolaños. 1992. Crustáceos decápodos bentónicos del monumento natural de Laguna de las Marites, Isla de Margarita, Venezuela. Boletín del Instituto Oceanográfico de Venezuela 38(2): 25-32.

Hernández-Ávila I, A Gómez, C Lira \& L Galindo. 2007. Benthic decapod crustaceans (Decapoda: Crustacea) of Cubagua Island, Venezuela. Zootaxa 1557: 33-45.

Marcano J \& J Bolaños. 2001. Cangrejos májidos (Decapoda: Brachyura: Majidae) de las aguas someras marinas de Venezuela. Boletín del Instituto Oceanográfico de Venezuela 40(1-2): 71-82.

Melo GA. 1996. Manual de indentificaçao dos Brachyura (Caranguejos e Siris) do litoral brasileiro, 603 pp. Plêiade, Sâo Paulo.

Ng P, D Guinot \& P Davie. 2008. Systema Brachyororum: Part I. An annotated checklist of extant brachyuran crabs of the world. The Raffles Bulletin of Zoology, Supplement 17: 1286.

Rodríguez G. 1980. Crustáceos decápodos de Venezuela, 444 pp. Instituto Venezolano de Investigaciones Científicas, Caracas.

Rathbun M. 1925. The spider crabs of America. Bulletin of United States National Museum 129: 1-613.

Stimpson W. 1871. Part I. Brachyura. In: de Pourtales LF (ed). Preliminary report on the Crustacea dredged in the Gulf Stream in the Straits of Florida. Bulletin of Museum of Comparative Zoology at Harvard College 2(2): 150-156.

Williams A. 1965. Marine decapod crustaceans of the Carolinas. Fishery Bulletin 65: 1-298.

Williams A. 1984. Shrimps, lobsters and crabs of the Atlantic coast of the Eastern United States, Maine to Florida, 550 pp. Smithsonian Institution Press, Washington. 